\title{
SARS-CoV-2 vaccination in patients with solid tumors or hematological malignancies: Is the pandemic over for fully vaccinated patients?
}

\author{
Niklas Zojer
}

Patients with cancer were reported to have an elevated risk of acquiring SARS-CoV-2 (severe acquired respiratory syndrome coronavirus 2) infection [1], possibly indicating an increased susceptibility to the virus, and have a high risk of developing severe COVID19 (coronavirus disease 2019), as well as dying from the disease. In a large study from the US [2], hospitalization for COVID-19 was necessary in $47.46 \%$ of oncologic patients, who had acquired the disease, and an overall mortality of $14.93 \%$ was seen, while numbers for patients without cancer were $24.26 \%$ and $5.26 \%$, respectively. A 30 -day mortality rate of $30 \%$ was found in a meta-analysis of cohort studies for patients with a diagnosis of cancer who were hospitalized for COVID-19 [3]. Available data point to certain subgroups of cancer patients being especially vulnerable, such as patients with hematological malignancies, most notably patients with leukemia and lymphoma being under active treatment, but also patients with lung cancer [4]. Delayed or absent viral clearance has been repeatedly observed in COVID-19 survivors with severe immune suppression [5]. More recently, the persistent immune dysregulation by SARS-CoV-2 infection in patients with hematological malignancies as compared to patients with solid tumors has been delineated by high throughput, high content flow-cytometry and multiplex cytokine analysis [6]. With regard to risk factors in the population of cancer patients, chemotherapy (i.e., cytostatic agents) was associated with a higher risk of mortality from COVID19 in some studies [4], but not in others [7]. Concerns were expressed that checkpoint inhibitors may predis-

\section{PD Dr. N. Zojer $(\bowtie)$}

1st Medical Department, Center for Oncology and

Hematology \& Palliative Care, Klinik Ottakring,

Montleartstraße 37, 1160 Vienna, Austria

niklas.zojer@gesundheitsverbund.at pose cancer patients to severe COVID-19, due to therapeutically activated T-cells augmenting the virally induced cytokine storm, but so far these concerns could not be substantiated. As in the general population, mortality of COVID-19 in cancer patients increases substantially with age. Currently, there are insufficient data for estimating COVID-19 risks for cancer survivors, i.e., patients in long-term follow-up without active disease or treatment, but highest risk for severe COVID-19 seems to affect cancer patients with active and/or advanced disease [1].

Clearly, the availability of SARS-CoV-2 vaccines has alleviated the immediate threat of the pandemic for cancer patients. However, several points need to be addressed in vaccination programs for these patients. Most available data in relation to vaccination efficacy in cancer patients relate to mRNA vaccines, either BNT162b2 or mRNA-1273. No safety issues distinct from those evident in the general population have appeared [8]. With regard to efficacy, a single vaccine dose seems unable to induce protective immunity in most cancer patients (immunity in less than $40 \%$ ), while this can be achieved in $94 \%$ of healthy controls [9]. After the second dose, response improves, with $98 \%$ of patients with solid tumors achieving seroconversion [10,11], approaching seroconversion rates of healthy subjects. On average, however, lower IgG titers are observed in cancer patients even after the full prime-boost scheme as compared to a healthy control group, with substantial heterogeneity [12, 13]. As antibody levels seem to wane over time, this heterogeneity likely has an impact on the duration of protection against infection and protection against developing severe disease. Possibly, earlier second boost vaccinations are necessary in patients with suboptimal antibody response, especially in the context of emergence of SARS-CoV-2 variants of concern, when low IgG levels unfavorably combine with low affinity to a mutated 
spike protein. While in a small study on healthy subjects protective antibodies persisted 6 months after the second dose of a mRNA vaccine, although at declining levels [14], in a larger trial on patients recovering from COVID-19, different trajectories of antibody decay were evident, with a significant fraction of patients losing protective titers within 6 months (27\%; [15]). Due to the short incubation period after SARSCoV-2 infection, it is unclear to what extent immunological memory (e.g., long-lived B-cells) confers some protection despite loss of anti-spike antibodies. Cytotoxic $\mathrm{T}$ cells are thought to rather control and clear established infection, rather than to prevent infection [16] and there seems to be a broad consensus with regard to the importance of humoral immunity in vaccine efficacy. However, different methods have been applied for measuring serological response to vaccination (receptor binding domain ELISA, pseudovirus or live virus neutralization assays), without clear indication which of these assays best correlates with clinical protection.

While immune responses to SARS-CoV-2 vaccines may be suboptimal in patients with solid tumors, antibody production is even more suppressed in patients with hematological malignancies. Seroconversion rates are around $80 \%$ in these patients after the second vaccine dose, lower than the $98 \%$ seroconversion rate noted in patients with solid tumors [10, 11]. In a study on 167 patients with chronic lymphocytic leukemia, the antibody response rate was only $39.5 \%$ after 2 doses of the BNT162b2 vaccine [17]. Vaccine response was especially poor in patients treated with either Bruton's tyrosine kinase (BTK) inhibitors or venetoclax and/or anti-CD20 antibodies (around $15 \%)$. None of the patients exposed to anti-CD20 antibodies < 12 months before vaccination responded. In a study on 320 myeloma patients, $84.2 \%$ of patients developed measurable anti-spike antibodies after full vaccination (with either BNT162b2 or mRNA1273), with titers varying three orders of magnitude [18]. In multivariate analysis, receiving either antiCD38 or BCMA-targeted treatment were significantly associated with a failure of vaccine response.

A large nation-wide cohort study from Lithuania can be highlighted, which included 885 patient with hematological malignancies, vaccinated with BTN162b2 [19]. A comparison was made between 315 of these patients and 67 age-matched healthcare workers, showing lower median anti-spike IgG responses in the group of patients with hematological malignancies. Particularly poor responses were evident in patients under treatment with BTK inhibitors, venetoclax or anti-CD20 therapy. Most notably, 9 SARS-CoV-2 infections and 3 COVID-19related deaths were observed in fully vaccinated patients with hematological malignancies, underlining the clinical significance of the serological findings. In contrast, more adequate immune responses were observed in patients vaccinated after autologous or allo- geneic transplantation (recommendation $>3$ months after transplantation) and in patients treated with imatinib, dasatinib, nilotinib, gilteritinib.

It was suggested that vulnerable individuals should receive the second vaccine dose in appropriate time, so that protective immunity is achieved as soon as possible. In addition, SARS-CoV-2 antibody levels should be determined in cancer patients to assure that protective levels of neutralizing antibodies have been reached. If that is not the case, a third booster dose might be administered. Eventually, a heterologous prime-boost regimen could be considered in these patients [20]. It seems important to provide a status of herd immunity in healthcare personnel, close family and other care givers. In care homes and hospitals for the elderly, influenza vaccination of care givers was shown to reduce mortality of patients and elderly residents significantly during the seasonal influenza epidemic [21, 22].

Two antibody cocktails are currently approved for treatment of vulnerable patients with early COVID-19 disease, to prevent disease deterioration. Studies in cancer patients are not available as yet, but likely these treatments are also beneficial in vulnerable cancer patients, i.e., those patients who are unable to mount an effective immune response to SARS-CoV-2 vaccination. Application could be considered as post-exposure prophylaxis or in early phases of COVID-19 disease, ideally as soon as a positive result is evident by SARS-CoV-2 PCR. Similar considerations might apply to pre-emptive therapy with convalescent plasma. In a randomized trial of convalescent plasma with high IgG titers, which included elderly patients within $72 \mathrm{~h}$ after the onset of mild COVID-19 symptoms [23], the risk to develop severe disease was reduced from $31 \%$ in the control population to $16 \%$ in the population receiving convalescent plasma.

Conflict of interest N.Zojer declares that he has no competing interests.

\section{References}

1. Liang W, et al. Cancer patients in SARS-CoV-2 infection: a nationwide analysis in China. Lancet Oncol. 2020;21(3):335-7.

2. WangQ, etal. Analyses of risk, racialdisparity, and outcomes among US patients with cancer and COVID-19 infection. JAMAOncol. 2021;7(2):220-7.

3. Desai A, et al. Mortality in hospitalized patients with cancer and coronavirus disease 2019: a systematic review and meta-analysis of cohort studies. Cancer. 2021;127(9):1459-68.

4. Lee LYW, et al. COVID-19 prevalence and mortality in patients with cancer and the effect of primary tumour subtype and patient demographics: a prospective cohort study. Lancet Oncol. 2020;21(10):1309-16.

5. Avanzato, et al. Case study: prolonged infectious SARScoV-2 shedding from an asymptomatic immunocompromised individual with cancer. Cell. 2020;183(7):19011912.e9. 
6. Abdul-Jawad S, et al. Acute immune signatures and their legacies in severe acute respiratory syndrome Coronavirus- 2 infected cancer patients. Cancer Cell. 2021;39(2):257-275.e6.

7. Jee J, et al. Chemotherapy and COVID-19 outcomes in patients with cancer. JClin Oncol. 2020;38(30):3538-46.

8. Goshen-Lago T, et al. Serologic status and toxic effects of the SARS-coV-2 BNT162b2 vaccine in patients undergoing treatment for cancer. JAMA Oncol. 2021; https://doi.org/ 10.1001/jamaoncol.2021.2675.

9. Monin L, et al. Safety and immunogenicity of one versus two doses of the COVID-19 vaccine BNT162b2 for patients with cancer: interim analysis of a prospective observational study. Lancet Oncol. 2021;22(6):738-9.

10. Addeo $\mathrm{A}$, et al. Immunogenicity of SARS-CoV-2 messenger RNA vaccines in patients with cancer. Cancer Cell. 2021;39:1-8.

11. Thakkar A, et al. Seroconversion rates following COVID19 vaccination among patients with cancer. Cancer Cell. 2021;39:1-10.

12. Massarweh A, et al. Evaluation of seropositivity following BNT162b2 messenger RNA vaccination for SARS-CoV-2 in patients undergoing treatment for cancer. JAMA Oncol. 2021;28:e212155. https://doi.org/10.1001/jamaoncol. 2021.2155.

13. Barriere J, et al. Impaired immunogenicity of BNT162b2 anti-SARS-CoV-2 vaccine in patients treated for solid tumors. Ann Oncol. 2021;32(8):1053-5.

14. Doria-Rose N, etal. Antibody persistence through 6 months after the second dose of mRNA-1273 vaccine for Covid-19. NEnglJ Med. 2021;384(23):2259-61.

15. Chia WN, et al. Dynamics of SARS-CoV-2 neutralising antibody responses and duration of immunity: a longitudinal study. Lancet Microbe. 2021;2(6):e240-e9.

16. Pollard AJ, Bijker EM. A guide to vaccinology: from basic principles to new developments. Nat Rev Immunol. 2021;21(2):83-100.
17. Herishanu Y, et al. Efficacy of the BNT162b2 mRNA COVID19 vaccine in patients with chronic lymphocytic leukemia. Blood. 2021;137(23):3165-73.

18. Van Oekelen A, et al. Highly variable SARS-CoV-2 spike antibody responses to two doses of COVID-19 RNA vaccination in patients with multiple myeloma. Cancer Cell. 2021;39:1-3.

19. Maneikis K, etal. Immunogenicity of theBNT162b2 COVID19 mRNA vaccine and early clinical outcomes in patients with haematological malignancies in Lithuania: a national prospective cohort study. Lancet Haematol. 2021. https:// doi.org/10.1016/S2352-3026(21)00169-1

20. Hill JA, et al. Immunogenicity of a heterologous COVID19 vaccine after failed vaccination in a lymphoma patient. Cancer Cell. 2021;39:1-2.

21. Carman WF, et al. Effects of influenza vaccination of health-care workers on mortality of elderly people in long-term care: a randomised controlled trial. Lancet. 2000;355(9198):93-7.

22. Hayward AC, et al. Effectiveness of an influenza vaccine programme for care home staff to prevent death, morbidity, andhealth serviceuseamongresidents: clusterrandomised controlled trial. BMJ. 2006;333(7581):1241.

23. Libster R, et al. Early high-titer plasma therapy to prevent severe Covid-19 in older adults. N Engl J Med. 2021;384(7):610-8.

Publisher's Note Springer Nature remains neutral with regard to jurisdictional claims in published maps and institutional affiliations.

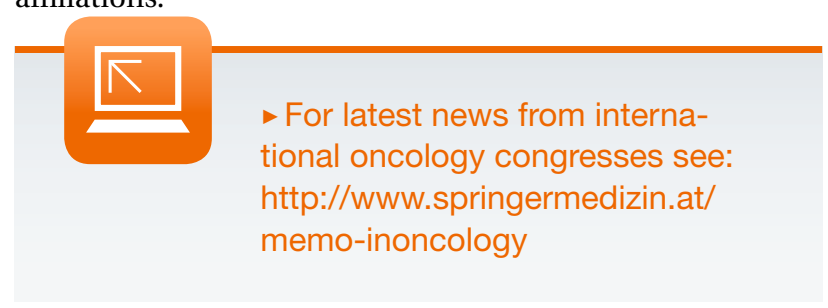

